\title{
Sodium antiporters of Pseudomonas aeruginosa in challenging conditions: effects on growth, biofilm formation, and swarming motility
}

\author{
Carla B. Schubiger ${ }^{1 \dagger}$, Kelli H. T. Hoang ${ }^{1,2^{*}+}$ and Claudia C. Häse ${ }^{1 *}$
}

\begin{abstract}
Background: Pseudomonas aeruginosa is a bacterial pathogen that can cause grave and sometimes chronic infections in patients with weakened immune systems and cystic fibrosis. It is expected that sodium/proton transporters in the cellular membrane are crucial for the organism's survival and growth under certain conditions, since many cellular processes rely on the maintenance of $\mathrm{Na}^{+}$and $\mathrm{H}^{+}$transmembrane gradients.

Results: This study focused on the role of the primary and secondary proton and/or sodium pumps Mrp, Nuo, $\mathrm{NhaB}, \mathrm{NhaP}$, and NQR for growth, biofilm formation, and swarming motility in $P$. aeruginosa. Using mutants with gene deletions, we investigated the impact of each sodium pump's absence on the overall growth, biofilm formation, motility, and weak acid tolerance of the organism. We found that the absence of some, but not all, of the sodium pumps have a deleterious effect on the different phenotypes of $P$. aeruginosa.

Conclusion: The absence of the Mrp sodium/proton antiporter was clearly important in the organism's ability to survive and function in environments of higher $\mathrm{pH}$ and sodium concentrations, while the absence of Complex I, which is encoded by the nuo genes, had some consistent impact on the organism's growth regardless of the pH and sodium concentration of the environment.
\end{abstract}

Keywords: Pseudomonas aeruginosa, Sodium, Antiporter, pH, Growth, Motility

\section{Background}

Pseudomonas aeruginosa is a ubiquitous opportunistic pathogen that can cause severe acute to chronic infections in humans. Among many other targets, it affects the skin, lungs, sensory organs, and urinary tract [15]. It is particularly devastating as a hospital-acquired infection and thrives in a variety of environments due to a large number of virulence factors and survival strategies. Besides the ability to quickly develop multidrug resistance, it also forms biofilms which make the bacteria more persistent and more resilient to treatment strategies $[8,11]$. P. aeruginosa is one of the leading problems in hospital-acquired infections, cystic fibrosis, and

\footnotetext{
* Correspondence: hoangk@oregonstate.edu; Claudia.hase@oregonstate.edu ${ }^{\dagger}$ Carla B. Schubiger and Kelli H. T. Hoang contributed equally to this work. 'Department of Biomedical Sciences, Carlson College of Veterinary Medicine, Oregon State University, Corvallis, OR 97331, USA

Full list of author information is available at the end of the article
}

immunocompromised patients [29]. In order to survive in these diverse environments, $P$. aeruginosa is equipped with a variety of cation transporters in the cellular membrane, which likely are essential to its physiology. Besides cation transport, some of the transporters import nutrients like amino acids and sugars, maintain the intracellular $\mathrm{pH}$, and are key to flagellar movement, which enables $P$. aeruginosa to move quickly through liquid or soft material and across semi-solid surfaces in a swimming or swarming motion $[39,46]$.

The cation pumps investigated in this work are the Multiple Resistance and $\mathrm{pH}$ antiporter (Mrp, also called Sha), Complex I encoded by the nuo gene cluster, and the NADH-ubiquinone oxidoreductase NQR, as well as the sodium/proton antiporters $\mathrm{NhaB}$ and NhaP. NQR and Complex I are generally regarded as primary pumps that expel sodium or protons from the cytoplasm to the 
periplasm by directly using metabolic energy and are generally regarded crucial for the establishment of an electrochemical membrane cation gradient. NQR is a respiration-linked protein that couples NADH oxidation with sodium transport in many species [40], including Vibrio cholerae [3, 18], V. alginolyticus [4, 19], V. harveyi [51], Haemophilus influenzae [20], and Klebsiella pneumonia [6]. Similarly, Complex I couples NADH oxidation with proton transport and is confirmed to also transport sodium in K. pneumoniae and Escherichia coli $[16,44]$. NQR and Complex I both contribute to the electron transport chain of $P$. aeruginosa, thus contributing to ATP generation and are possibly important to energy production and the maintenance of the electrochemical gradient $[2,50]$. Just recently, however, Raba et al. [37] suggested that Pa-NQR is not a sodium pump, but acts as a $\mathrm{H}^{+}-\mathrm{NQR}$. Therefore, our study will seek to confirm this new finding and will also investigate the sodium transporting involvement of $P$. aeruginosa Complex I.

Secondary sodium transporters utilize $\Delta \mathrm{pH}$, the electrochemical proton gradient, to expel sodium in exchange for protons [22]. Interestingly, the most intensely studied and widely present antiporter NhaA is missing in $P$. aeruginosa. However, it expresses $\mathrm{NhaP}$ and $\mathrm{NhaB}$ which are single subunit transporters [28]. NhaP of $P$. aeruginosa is able to export sodium in exchange for protons but cannot transport lithium [47], while $\mathrm{Pa}$ NhaB can export sodium and lithium and does so preferentially at alkaline conditions [28]. In contrast to NhaP and NhaB, Pa-Mrp, also termed Sha in PA01 [26], is composed of multiple units (mrpA'CDEFG) and also facilitates sodium export and $\mathrm{pH}$ homeostasis at alkaline $\mathrm{pH}$, with potential functions beyond cation transport. Interestingly, Pa-Mrp contributed to pathogenicity when assayed in mice, but had no influence in antibiotic resistance [26]. Overall, Mrp has also been shown to confer bile acid resistance when expressed in Escherichia coli [13] and Vibrio cholerae [13, 22], and played a role in nitrogen metabolism, cell motility and biofilm formation in $V$. cholerae [1]. In addition, putative $m r p$ operons have been found in a large variety of pathogens and environmental strains that often survive in very extreme conditions [45].

In addition to growth in challenging environmental conditions, this study also investigates the contributions of these primary and secondary membrane transporters to biofilm formation and swarming motility. Swarming motility is the rapid, coordinated, and grouped movement of bacteria over a semi-solid surface [39]. Flagellar movement, which in $P$. aeruginosa enables swimming motility, also assists in swarming motility and is powered by the proton motive force $[12,42]$. This force is generated by the transmembrane proton concentration gradient, created and maintained by primary or secondary proton pumps [12, 32]. Clearly, membrane cation transporters contribute to various cellular functions and a deeper understanding of these pumps is needed as they are opportunities for new drug targets $[9,10]$.

The aims of this study are to assess the role of certain transporters of $P$. aeruginosa (Fig. 1 ) in different $\mathrm{pH}$ and sodium environments in regards to growth, biofilm formation, motility at $\mathrm{pH} 6.5,7.5$, and 8.5 , and sodium concentrations up to $500 \mathrm{mM}$ and also to assess their sensitivity to weak acids.

\section{Results}

\section{Sodium and $\mathrm{pH}$ tolerance}

All $P$. aeruginosa strains listed in Table 1 were grown at $\mathrm{pH} 6.5,7.5$, and 8.5 in $\mathrm{LBB}^{-}$with no added sodium or added sodium ranging from 100 to $500 \mathrm{mM}$. While hourly measurements were taken, only time points 6 and $18 \mathrm{~h}$ are presented for better clarity of the graphs and the statistical analyses; this selection was arbitrary but the time points were representative of early exponential and early stationary phase, respectively. The full 24-h growth curves are available as supplemental data S1-3. Generally, strains grew best at $\mathrm{pH} 6.5$ and worst at $\mathrm{pH}$ 8.5. While higher sodium conditions were disadvantageous for all strains in early exponential phase (Fig. 2ac), those differences were less obvious at early stationary phase (Fig. $2 \mathrm{~d}-\mathrm{f}$ ). In addition, variability between the

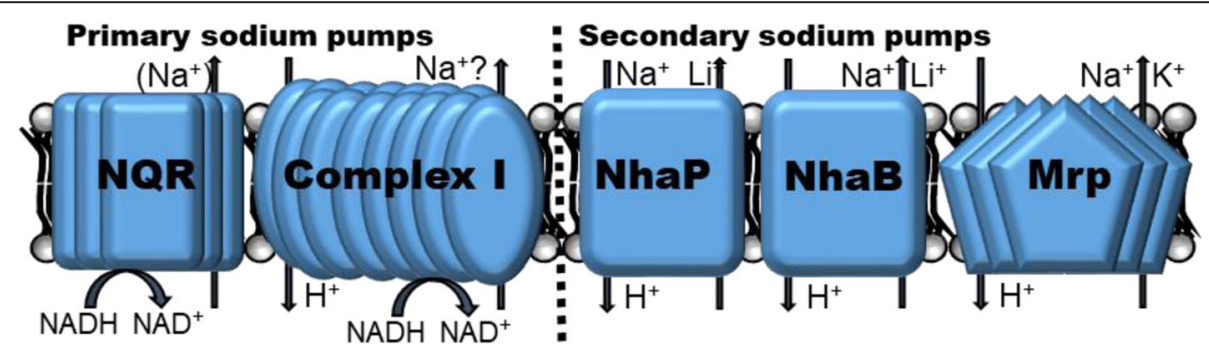

Fig. 1 Primary and secondary sodium antiporter discussed in this article. NQR (NADH-ubiquinone oxidoreductase) is a major primary sodium pump in many species, but likely not $P$. aeruginosa. Complex I's involvement as a primary sodium pump is questionable in $P$. aeruginosa. NhaP and NhaB are commonly transporting sodium and lithium and Mrp is a powerful transporter of sodium and potassium 
Table 1 Strains used in this work. All strains were obtained from the P. aeruginosa PA14 transposon insertion library. The location of the insertion in the multi-subunit proteins Mrp, NQR and Nuo, is subunit A for Mrp and NQR, and subunit NuoB or 1 in Complex I. Two different mutants were available for NhaP, and labeled mutant nhaP*1 (insertion at base pair 50) and mutant nhaP*2 (insertion at base pair 356), respectively. Both NhaP mutants were included in our testing

\begin{tabular}{|l|l|}
\hline $\begin{array}{l}\text { P. aeruginosa } \\
\text { PA14 Strain }\end{array}$ & Description \\
\hline WT & Wild-type \\
\hline mutant $m r p A$ & Transposon insertion in subunit MrpA \\
\hline mutant $n q r A$ & Transposon insertion in subunit NqrA \\
\hline mutant $n h a B$ & Transposon insertion in NhaB \\
\hline mutant $n u o B$ & Transposon insertion in subunit NuoB \\
\hline mutant $n h a P^{* 1}$ & Transposon insertion at base pair 50 of NhaP \\
\hline mutant $n h a P^{* 2}$ & Transposon insertion at base pair 356 of NhaP \\
\hline
\end{tabular}

biological replicates increased with increasing incubation length, which was likely related to exhaustion of the growth medium and were most severe at $24 \mathrm{~h}$ (Fig. $2 \mathrm{~d}-\mathrm{f}$; S1-3). At acidic conditions, none of the mutations resulted in an increase in sodium sensitivity when compared to the wild-type strain, regardless of growth phase (Fig. 2a-d). At pH 7.5 and in exponential phase, the MrpA mutant was highly growth retarded in a sodium concentrations of $200 \mathrm{mM}$ and higher (Fig. 2b, S2) and approached wild-type levels in the 200 and $300 \mathrm{mM}$ condition after $18 \mathrm{~h}$, but the strain was still highly growth deficient at sodium concentrations of 400 and $500 \mathrm{mM}$ (Fig. 2e). At pH 8.5, growth was essentially arrested with the addition of $\geq 100 \mathrm{mM} \mathrm{NaCl}$ (Fig. 2c, f), with the exception that in $100 \mathrm{mM}$ sodium, growth started to recover after $14 \mathrm{~h}$, although the growth retardation continued to be statistically significant when compared to the wild-type strain (S3). The asterisks in the graphs indicate statistical significance $(p<0.0001 ;$ Fig. 2$)$. It should be noted that the Complex I mutant often displayed less growth compare to the wild-type, but the differences were small and somewhat inconsistent.

\section{Biofilm assays}

To account for growth differences, a Biofilm Index was calculated as a ratio of OD570nm/595 nm (optical density at $570 \mathrm{~nm}$-crystal violet staining divided by optical density of the culture in each respective well measured at $595 \mathrm{~nm}$ before the wash and staining steps). A higher index means more proportional biofilm production relative to growth. Biofilm formation at all sodium concentrations was overall the lowest at $\mathrm{pH}$ 8.5 (Fig. 3c). Within $\mathrm{pH} 8.5$, highest Biofilm Index was calculated when $500 \mathrm{mM}$ sodium chloride was added (Fig. 3c). Within the measurements at pH 6.5 and 7.5 , slightly higher Biofilm Indexes were seen when no sodium was supplemented $(\mathrm{pH} 7.5)$ and at
pH 6.5 with the addition $500 \mathrm{mM}$ sodium chloride (Fig. 3a, b). Sodium additions ranging between 100 and $400 \mathrm{mM}$ resulted in fairly similar Biofilm Indexes at $\mathrm{pH} 6.5$ and 7.5 (Fig. 3a, b). At pH 6.5, there were no statistical significant differences between the wildtype and the mutant strains at any of the sodium conditions evaluated (Fig. 3a). The MrpA mutant displayed a severely heightened Biofilm Index in compare to the wild-type strain when 400 and $500 \mathrm{mM}$ $\mathrm{NaCl}$ was added at $\mathrm{pH} 7.5$, while at $\mathrm{pH} 8.5$ the addition of $\geq 200 \mathrm{mM} \mathrm{NaCl}$ caused significantly higher Biofilm Indexes with a $p$ value of $<0.001$ (Fig. 3b, c).

\section{Swarming motility}

Swarming motility was assessed quantitatively by measuring the area of growth across the semi-solid surface after $18 \mathrm{~h}$ of incubation (Fig. 5). Preliminary experiments revealed that swarming motility was not affected by the mutations within NQR, NhaB, and NhaP (data not shown); thus, these experiments only compared the mutants of Complex I and Mrp to the wild-type strain (Fig. 5). Overall growth areas were the largest at $\mathrm{pH} 7.5$ (Fig. 4b) and smallest at pH 8.5 (Fig. 4c). Sodium addition or mutation did not significantly affect the swarming motility at pH 6.5 (Fig. 4a). At pH 7.5 and no added sodium, the Complex I mutant showed significantly $(p<0.0001)$ less swarming thus covering less area on the agar plate (Fig. 4b). While when $400 \mathrm{mM} \mathrm{NaCl}$ was added, the Mrp mutant displayed less motility $(p<$ 0.0001; Fig. 4b). At alkaline $\mathrm{pH}$, both mutants were significantly different in compare to the wild-type (Fig. 4c). The Mrp mutant did not grow when $400 \mathrm{mM} \mathrm{NaCl}$ was added $(p<0.0001)$ (Fig. 5), and the Complex I mutant became hypermotile when no additional sodium was added $(p<0.0001)$ but also displayed significantly reduced swarming motility with the addition of $400 \mathrm{mM}$ $\mathrm{NaCl}(p<0.0001 ;$ Fig. 4c). 


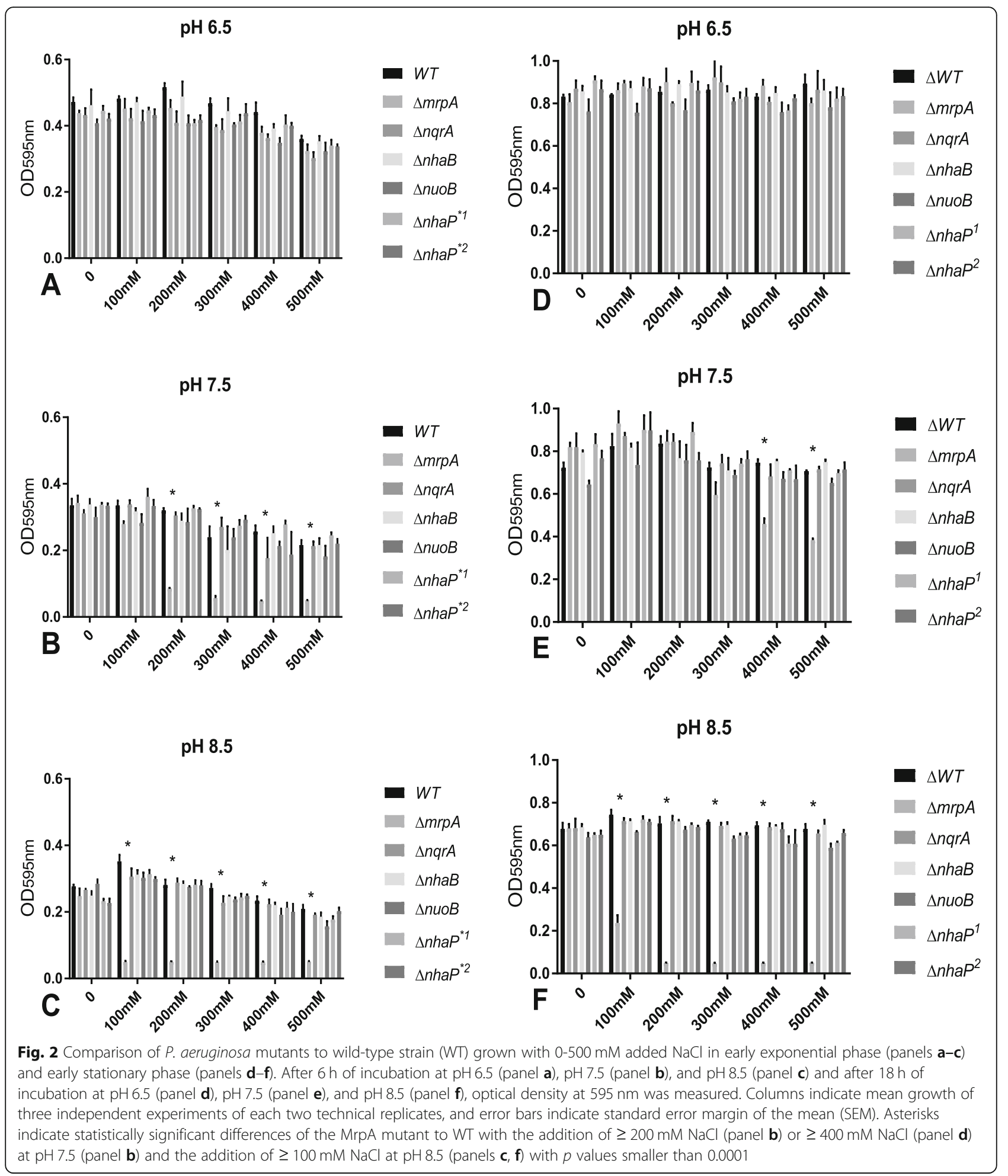

\section{Weak acid resistance}

Our previously published work on Mrp in $V$. cholerae [1] identified susceptibility of the Mrp mutant to three weak acids: docusate, also known as dioctyl sulfosuccinate, $n$-lauroylsarcosine, and probenecid. Therefore, those weak acids were tested in this assay. Due to the structural similarities of Mrp to Complex I (reviewed in the discussion), we tested both mutants and compared them to the wild-type strain. Both mutants did not reveal any significant differences compared to the wild- 
pH 6.5

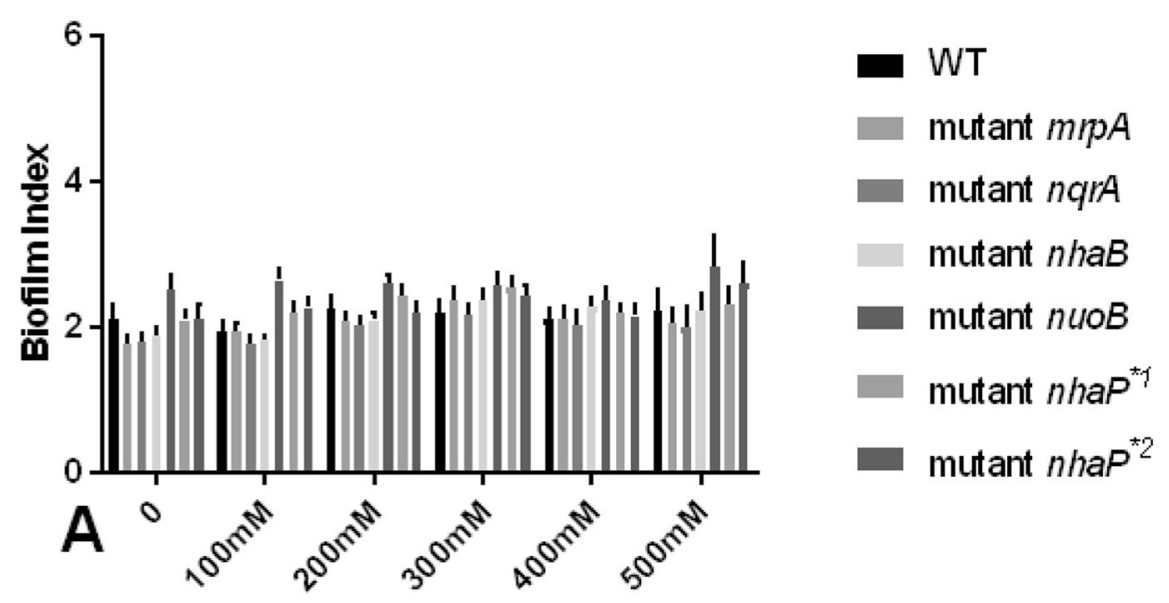

pH 7.5

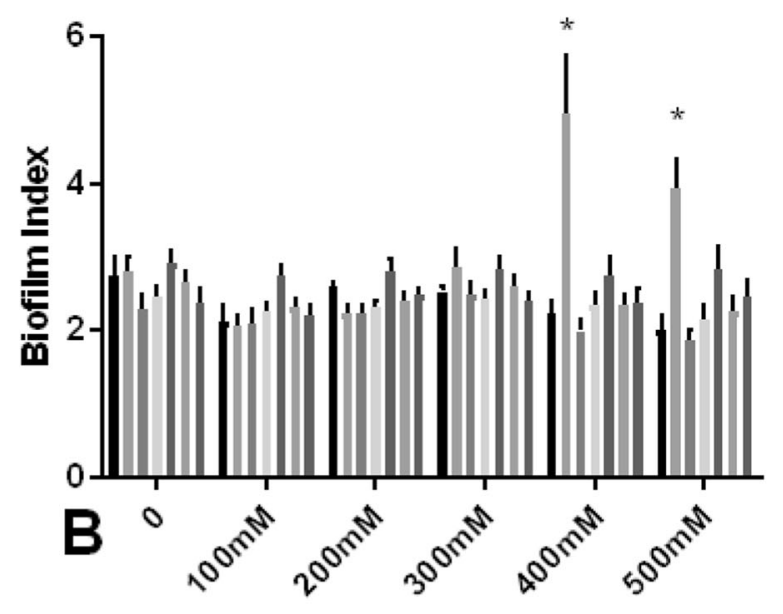

WT

- mutant mrpA

- mutant nqra

- mutant $n$ haB

- mutant $n U O B$

- mutant nhaP ${ }^{*}$

- mutant nhaP ${ }^{\text {22 }}$

pH 8.5

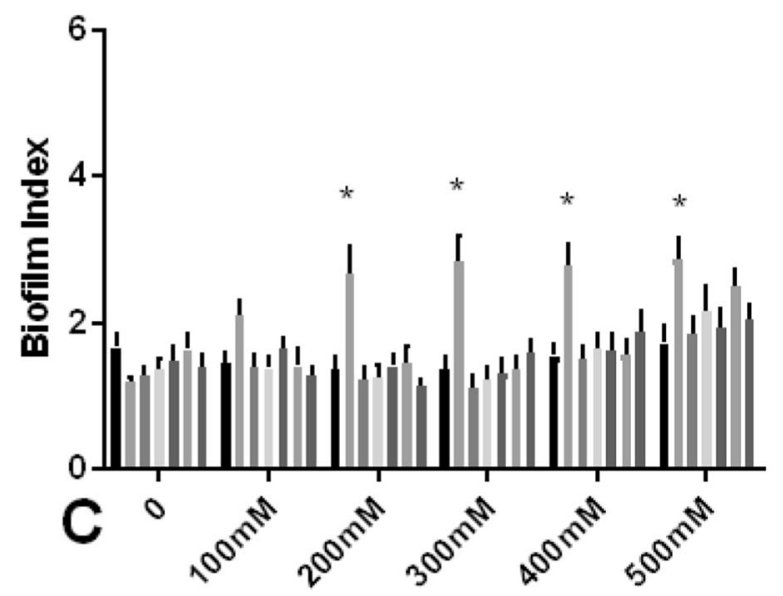

- WT

- mutant mrpA

- mutant $n q r A$

- mutant $n h a B$

- mutant $n u \circ B$

mutant nhaP ${ }^{*}$

mutant $n h a P^{* 2}$

Fig. 3 (See legend on next page.) 
(See figure on previous page.)

Fig. 3 Biofilm index comparison of $P$. aeruginosa mutants to wild-type strain (WT) grown with $0-500 \mathrm{mM}$ added $\mathrm{NaCl}$ in stationary phase. After $24 \mathrm{~h}$ of incubation at pH 6.5 (panel a), pH 7.5 (panel b), and pH 8.5 (panel c), optical density of the cultures in the wells was measured at $595 \mathrm{~nm}$. Then, the biofilms in the wells was stained and the wells measured at $570 \mathrm{~nm}$. The biofilm index was calculated as OD570/OD595 to account for growth differences. Columns indicate mean ratio of five independent experiments, and error bars indicate standard error margins of the mean (SEM). Asterisks indicate statistically significant differences in Biofilm Index of the MrpA mutant with the addition of $\geq 400 \mathrm{mM} \mathrm{NaCl}$ at pH 7.5 (panel b) or with the addition of $\geq 200 \mathrm{mM} \mathrm{NaCl}$ at pH 8.5 (panel c) to WT with $p$ values equal or smaller than 0.001

type strain or the results were inconclusive, making the involvement of these enzymes in drug transport questionable $(S 4,5,6)$.

\section{Discussion}

Sodium/proton antiporters are important membrane proteins that export sodium in exchange with protons for internal $\mathrm{pH}$ homeostasis and play roles in energy metabolism, nutrient acquisition, and more [35]. These transporter proteins can be found in a wide variety of eukaryotic and prokaryotic cells [35, 36, 49]. Loss of such proteins can have significant consequences to the cell thus making these proteins interesting drug target candidates. In this study, growth, biofilm formation, swarming motility, and resistance to weak acids under different environmental conditions were examined using transposon insertion mutants in $P$. aeruginosa. The primary transporters NQR and Complex I were studied, as well as the secondary antiporters Mrp, NhaB, and NhaP.

The loss of the NQR did not affect growth under challenging sodium and $\mathrm{pH}$ conditions, which confirms a recent study by Raba et al. [37] who suggested that in contrast to NQR in other species, such as Vibrio $[3,23$, 27, 51], Pa-NQR is a proton pump with no affinity for sodium. In addition to that, our results suggest that loss of NQR does not affect $\mathrm{pH}$ homeostasis, biofilm formation, or swarming motility, which might be due to complementary proton pumping activity of Complex I. This hypothesis should be tested with a double deletion mutant of nqr and nuo in the future.

NhaB is not essential in $V$. cholerae as it has a supporting function and is, when lost, easily replaced by other sodium transporters, such as NhaA or NhaD, which are missing in P. aeruginosa [21]. Based on our results $\mathrm{Pa}-\mathrm{NhaB}$ is unlikely a major contributor to sodium transport as its mutation did not interfere with growth, biofilm formation, or swarming motility at any of the tested conditions. In addition, it had been reported that when $\mathrm{Pa}-\mathrm{NhaB}$ was expressed in everted membrane vesicles at $\mathrm{pH} 8.0$ it showed a higher affinity to lithium than sodium [28]. This could suggest that $\mathrm{Pa}-$ $\mathrm{NhaB}$ has only a supportive function in sodium transport. To further investigate this theory, a triple deletion mutant strain lacking NhaA, NhaP, and Mrp should be constructed as these proteins can likely complement each other's sodium transport in single mutants.
Pa-NhaP has also only been assayed when expressed in everted membrane vesicles of the antiporter-deficient E. coli strain KNabc, where Pa-NhaP was found to be a major sodium but not lithium transporter [28]. While we did not evaluate NhaP in regards to lithium, we tested two independent $P$. aeruginosa NhaP mutants in different $\mathrm{pH}$ and sodium conditions and did not observe an increase of sodium sensitivity compared to the wildtype strain. Also unaffected were biofilm formation and swarming motility. This lack of effect is likely due to a concerted effort of other sodium pumps (such as Mrp) in challenging environmental conditions and should be further investigated in the future.

When the MrpA subunit was mutated, neither growth nor biofilm formation or swarming was affected at $\mathrm{pH}$ 6.5, but significant changes occurred at $\mathrm{pH} 7.5$ and particularly at $\mathrm{pH} 8.5$. At $\mathrm{pH} 7.5$ and early log phase, sodium concentrations of $\geq 200 \mathrm{mM}$ led to a prolonged lag phase, while at $\mathrm{pH} 8.5, \geq 200 \mathrm{mM} \mathrm{NaCl}$ resulted in a growth arrest from which the strain did not recover. An involvement of the Pa-mrp gene cluster (previously also designated sha [26];) as a major contributor to sodium transport at alkaline $\mathrm{pH}$ was previously demonstrated by the quenching method using everted membrane vesicles of the sodium sensitive $E$. coli TO114 which lacks three major $\mathrm{Na}^{+} / \mathrm{H}^{+}$antiporters [41] and by disruption of PA01-shaA [26]. In our experiments, the Pa-Mrp mutant experienced a severely prolonged lag phase when exposed to high concentrations of sodium (400 and 500 $\mathrm{mM} \mathrm{NaCl}$ ) at $\mathrm{pH} 7.5$ and a complete growth retardation for at least $24 \mathrm{~h}$ at $\mathrm{pH} 8.5$ when $\geq 200 \mathrm{mM} \mathrm{NaCl}$ was added, but showed successful formation of biofilms under those conditions. This could be explained by altered physiological characteristics often found when planktonic bacteria become sessile, such as a vastly altered gene expression profiles and slowed growth rate intended to make the sessile form more resilient to adverse environmental conditions (suggested by [11]). When biofilm formation is high, motility/swarming activity is usually low; this phenomena is a basic function in bacterial physiology and is antagonistically regulated $[5,48]$.

Swarming is the rapid multicellular movement of bacteria across an $>0.3 \%$ agar surface along a nutrient gradient generated by the bacteria, and the movement is powered by a rotating flagella [24]. While in some 


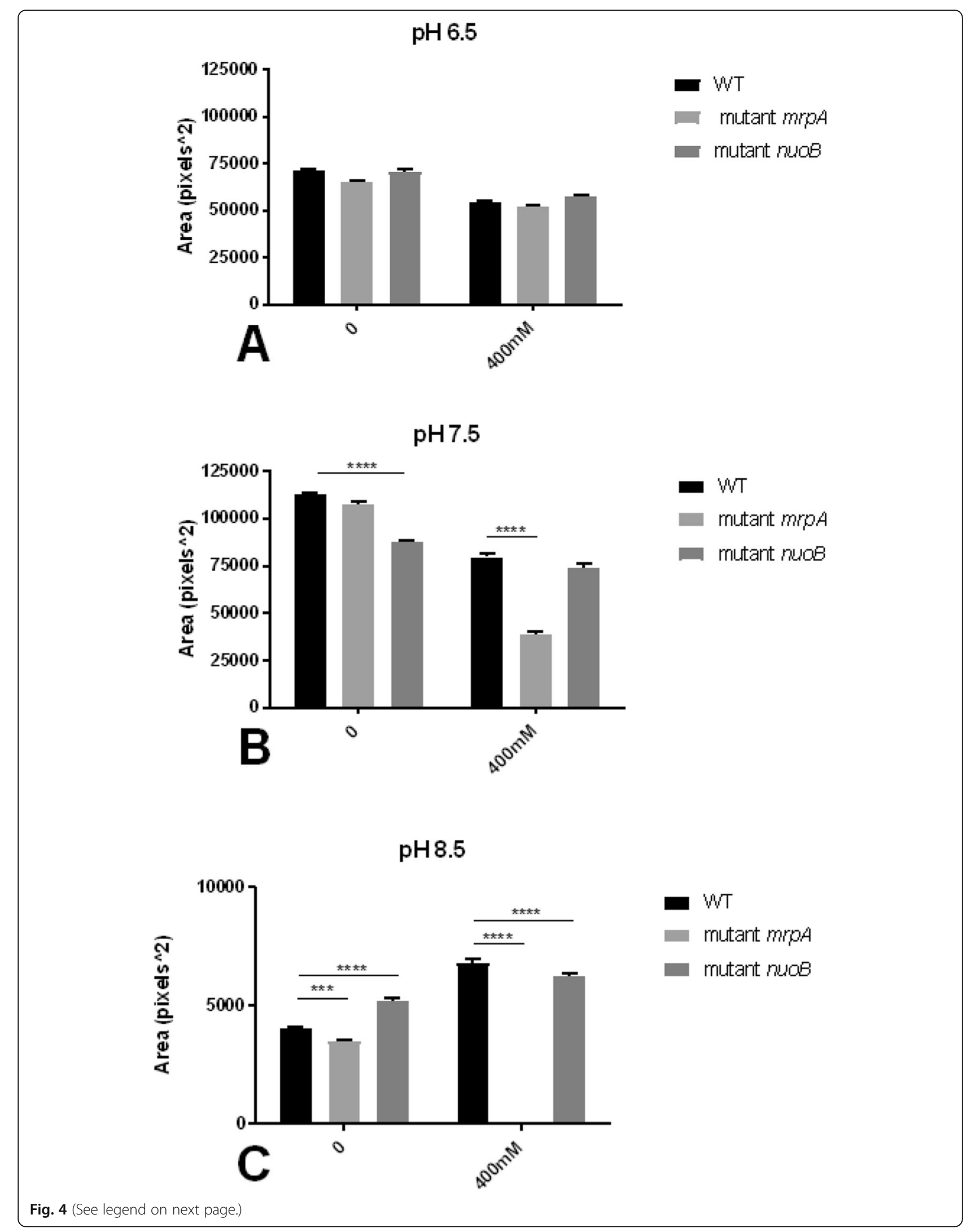


(See figure on previous page.)

Fig. 4 Swarming motility of the P. aeruginosa MrpA and Complex I mutants compared to the wild-type strain (WT). The swarming motility was accessed after $18 \mathrm{~h}$ on semi-solid agars of $\mathrm{pH} 6.5,7.5$, or 8.5 and no additional $\mathrm{NaCl}$ or when supplemented with $400 \mathrm{mM} \mathrm{NaCl}$. Swarming motility was measured as area of growth with the software ImageJ and graphed as square-pixels. Representative photographs are shown in Fig. 5. No statistical differences were observed at acidic pH (panel a). At pH 7.5 and without added sodium, the Complex I mutant showed significantly reduced swarming motility in compare to WT $(p<0.0001)$ while the Mrp mutant but not the Complex mutant showed hypomotility when 400 $\mathrm{mM} \mathrm{NaCl}$ was added (panel $\mathbf{b}$ ). Very little swarming motility was detected at pH 8.5 (panel $\mathbf{c}$ ), with significant hypermotility of the Complex I mutant when no sodium was added $p<0.0001)$, but reduced swarming of the Mrp mutant $(p<0.001)$. When at pH 8.5 (panel $\mathbf{c}$ ), $400 \mathrm{mM} \mathrm{NaCl}$ was added, the Mrp mutant did not grow on the semi-solid agar (see Fig. 5), and the Complex I mutant revealed significantly reduced swarming motility in compare to WT (both $p<0.0001$ )

bacteria, like $V$. cholerae, flagellar rotation is powered by sodium; this does not seem to be the case in P. aeruginosa $[5,12]$, which has a polar flagella driven by a dual set of motor proteins, MotA/B/Y and MotC/D [12]. Different to many other bacteria, swarming in $P$. aeruginosa seems to be more complex and in some capacity also supported by Type IV pili [25, 34]. Swarming is often defined as the surface-motility used to translocate cells to a more favorable environment [12]. It can therefore be assumed that lack of swarming in unfavorable environmental conditions might result in heightened biofilm formation; however, from our results we conclude that swarming, or lack-thereof, is a poor predictor of biofilm production in $P$. aeruginosa. Regardless of the generally

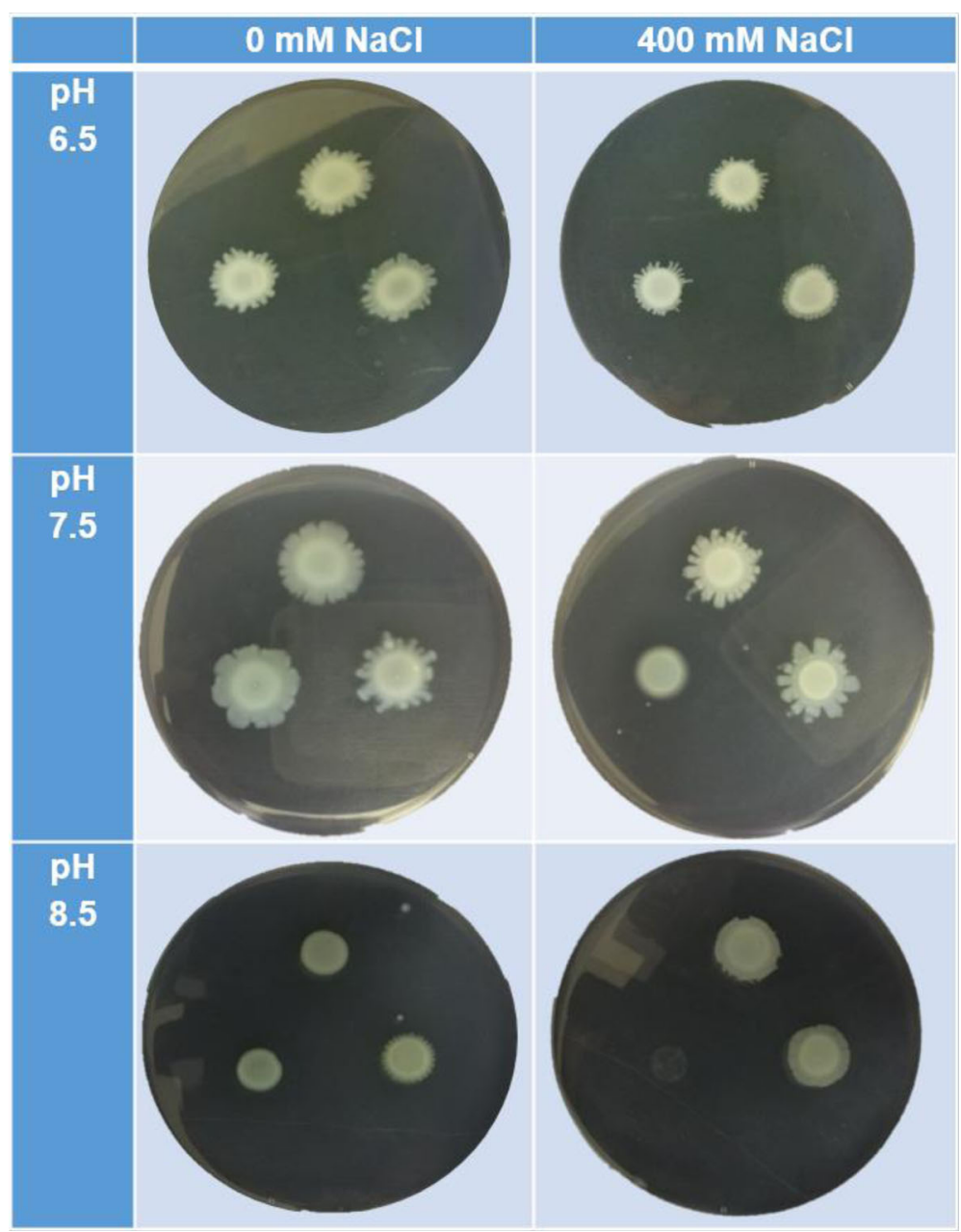

Fig. 5 Swarming motility of the P. aeruginosa wild-type (WT), MrpA mutant and Complex I mutant strains. Five microliters of culture was applied onto the semi-solid agar of pH 6.5 (upper row), 7.5 (middle row), and 8.5 (lowest row), and without ( $0 \mathrm{mM} \mathrm{NaCl}$, left column) or with $400 \mathrm{mM}$ $\mathrm{NaCl}$ (right column) and grown for $18 \mathrm{~h}$. In this figure are photographs of representative agar plates at each condition with three aliquots of bacterial culture arranged as top (WT), lower right (Complex I mutant), and lower left (MrpA mutant) 
favorable effects of sodium addition to growth of our tested strains at pH 7.5 and 8.5 (Fig. 2), the Complex I mutant showed significantly reduced swarming at $\mathrm{pH} 7.5$ and no added sodium (Fig. 4b; $p<0.0001$ ), where we would likely expect higher swarming to get the cells into a more sodium-rich environment. A possible explanation is that this transporter is a major contributor to the protein motive force and thus provides the power necessary for flagella rotation. However, generally, this mutation did not alter any other phenotypes tested in this study, suggesting that other proteins collaborate with Complex I to enable normal phenotypes. In contrast at $\mathrm{pH} 8.5$, the Complex I mutant revealed an increased swarming phenotype at the unfavorable sodium-depleted condition and reduced swarming in sodium-rich conditions, which supports the translocation to more favorable conditions idea (Fig. 4c; $p<0.0001$ ). Clearly, future studies are required to better understand these phenotypes.

The Mrp mutant showed significantly reduced swarming motility compared to the wild-type strain at $\mathrm{pH} 7.5$ and $400 \mathrm{mM}$ added sodium (Fig. 4b; $p<0.0001$ ). However, at these environmental conditions, the strain's growth was also significantly slowed and reduced compared to the wild-type strain (Fig. 2e), confirming Mrp's involvement in sodium transport. However, this reduced growth could also explain the quantitatively reduced, sodium-independent swarming motility (Fig. 4b). Overall, the Mrp protein becomes most relevant when sodium levels in the environment are elevated $(\geq 200 \mathrm{mM})$ and $\mathrm{pH}$ is 7.5, and it becomes essential at $\mathrm{pH} 8.5$ in the presence sodium. In addition at those conditions ( $\geq \mathrm{pH}$ 7.5 and $\geq 200 \mathrm{mM} \mathrm{NaCl}$ ), lack of a functional Mrp protein complex resulted in higher biofilm formation, even though at $\mathrm{pH} 8.5$ no growth and thus no swarming motility were evident. In summary, Mrp is clearly important to $P$. aeruginosa's survival in challenging environments.

Mrp and Complex I seem to have some evolutionary relationship based on the homology between MrpC and NuoK [31], MrpA and NuoL, and MrpD and NuoMN [33]. Interestingly, the NuoL subunit has been suggested to transport sodium in $E$. coli $[17,22,43]$. While not reported as statistically significant in this work due to our stringent $p$ value threshold and the randomness of the occurrences, a few differences between the wild-type and the NuoB mutant might be notable, for example at $\mathrm{pH}$ 8.5 , the growth of the NuoB mutant was slightly reduced when $500 \mathrm{mM} \mathrm{NaCl}$ was added $(p=0.0165)$, or slightly elevated Biofilm Indexes were seen at pH 6.5 and 100 $\mathrm{mM} \mathrm{NaCl}(p=0.0246)$, as well as at $\mathrm{pH} 7.5$ and $500 \mathrm{mM}$ $\mathrm{NaCl}(p=0.0323)$.

\section{Conclusion}

Antiporters are membrane proteins that facilitate a variety of tasks to enable survival in quickly changing, and often challenging environmental conditions. Such tasks include intracellular $\mathrm{pH}$ homeostasis and cation as well as nutrient transport across the cellular membrane. In addition, antiporters often also contribute to flagellar movements. We have found that in $P$. aeruginosa the antiporters NQR, NhaB, and NhaP have only subordinate roles in maintaining normal cell physiology when exposed to high sodium and/or high $\mathrm{pH}$ environments. In contrast, Complex I, while unlikely essential for sodium transport, seems to be involved in swarming ability, and most importantly, Mrp is indispensable for growth in challenging sodium and $\mathrm{pH}$ conditions.

\section{Methods}

All strains were originating from a $P$. aeruginosa strain PA14 transposon insertion library [30]. Due to different locations of the transposon insertion in NhaP, two different mutants of NhaP were evaluated (Table 1). Strains were isolated from the frozen stocks by streaking them on Luria-Berani (LB) agar and incubating them overnight at $37^{\circ} \mathrm{C}$. Fresh single colonies were cultured in LB Lennox broth $(5 \mathrm{~g}$ per $\mathrm{L}$ of $\mathrm{NaCl})$ in a roller drum or table top shaker at $7 \times g$ at $37^{\circ} \mathrm{C}$ overnight. For the growth curves, buffered $\mathrm{LBB}^{-}$(Luria broth without any additional sodium chloride; $10 \mathrm{~g}$ per $\mathrm{L}$ peptone, $5 \mathrm{~g}$ per $\mathrm{L}$ yeast extract, $60 \mathrm{mM}$ BIS-TRIS propane) was used and the residual amount of sodium in our $\mathrm{LBB}^{-}$was 13-19 $\mathrm{mM} \mathrm{Na}{ }^{+}$.

\section{Sodium and $\mathrm{pH}$ tolerance growth assays}

All wells of a 96-well flat-bottom cell culture plate (Greiner Bio-one) were filled with $140 \mu \mathrm{l}$ of $\mathrm{LBB}^{-}$media of $\mathrm{pH} 6.5$ and increasing sodium concentrations of 0,100 , $200,300,400$, and $500 \mathrm{mM}$ in technical duplicates throughout the 12 well columns. Overnight cultures of each strain were pelleted by centrifugation at $3800 \times g$ for 5 min, washed with $\mathrm{LBB}^{-}$, and re-suspended to an optical density of 0.1 measured by wavelength $600 \mathrm{~nm}$ $\left(\mathrm{OD}_{600}\right)$. Twenty microliters of each culture was transferred to their respective wells, while control wells received $20 \mu \mathrm{l}$ of sterile broth. All wells were then covered with $50 \mu$ of mineral oil to prevent evaporation without affecting growth [7] and incubated at $37^{\circ} \mathrm{C}$ and medium intensity shaking in a BioTek SynergyMx microplate reader for $24 \mathrm{~h}$. The $\mathrm{OD}_{595}$ was measured hourly for during that time. The plate setup and the measurements were repeated for $\mathrm{pH} 7.5$ and 8.5 and a total of three biological replicates per $\mathrm{pH}$.

\section{Biofilm assays}

For this assay, wells of a 96-well flat-bottom cell culture plate (Greiner Bio-one) were filled with $180 \mu \mathrm{l}$ of $\mathrm{pH}$ and sodium adjusted $\mathrm{LBB}^{-}$media. Again, the tested $\mathrm{pH}$ values were $6.5,7.5$, and 8.5 , and sodium concentrations 
ranged from zero to $500 \mathrm{mM} \mathrm{NaCl}$ added. Cultures were re-suspended to an $\mathrm{OD}_{600}$ of 0.1 and $20 \mu \mathrm{l}$ was added to each respective well in duplicates for each condition. Using an iMark plate reader (BioRad), the optical density at $\mathrm{OD}_{595}$ was recorded for each well after a 24-h incubation at $37^{\circ} \mathrm{C}$ (no shaking). Then, the media was discarded and the plates were washed three times with deionized water and left to dry at room temperature. One hundred microliters of an $0.1 \%(\mathrm{w} / \mathrm{v})$ crystal violet stain was added to each well and shaken at $7 \times g$ and $37^{\circ} \mathrm{C}$ in an orbital shaker (Thermo Scientific MaxQ 4000) for $30 \mathrm{~min}$. After staining, the plates were washed three times with deionized water and left to dry at room temperature. Then, $200 \mu \mathrm{l}$ of $95 \%$ ethanol was added to the wells to solubilize the stained biofilm and consequently incubated for $15 \mathrm{~min}$ at room temperature. Optical density at $570 \mathrm{~nm}$ was measured in the iMark plate reader to assess the biofilm formation, and the biofilm ratio $\left(\mathrm{OD}_{570} / \mathrm{OD}_{595}\right)$ was calculated to account for growth deficiencies [1]. These experiments were independently repeated five times for each $\mathrm{pH}$.

\section{Swarming motility}

LBB $^{-}$semi-solid agar plates $(0.5 \% \quad w / v$ agar $)$ were adjusted to $\mathrm{pH} 6.5,7.5$, or 8.5 , and supplemented with either 0 or $400 \mathrm{mM}$ sodium chloride. Overnight cultures of each strain were pelleted in a table top centrifuge at $3800 \times g$ for $5 \mathrm{~min}$ and re-suspended in $\mathrm{LBB}^{-}$media of $\mathrm{pH} 6.5,7.5$, or 8.5 to an $\mathrm{OD}_{600}$ of 0.3 . For each strain, $5 \mu \mathrm{l}$ of culture was delivered to the surface of the agar. The cultures were left to dry for $15 \mathrm{~min}$ at room temperature on the bench before being moved to the incubator where they were incubated at $37^{\circ} \mathrm{C}$ for $18 \mathrm{~h}$. All agar plates were photographed with 16 megapixel digital camera and the photographs analyzed using the freely available software Image J [38] and the ImageJ plugin Simple Interactive Object Extraction (SIOX) [14]. The experiment was independently repeated eight times, with two technical replicates per experiment.

\section{Weak acid resistance}

Since most statistically significant differences in growth and swarming motility were limited to the mutants $m r p A$ and $n u о B$, and only these two mutants were submitted to the weak acid resistance evaluation. Overnight cultures of the wild-type strain and the MrpA and NuoB mutant were pelleted in a tabletop centrifuge at $3800 \times g$ for $5 \mathrm{~min}$ and re-suspended with fresh LB media $(\mathrm{pH} \pm$ 7.0) to an $\mathrm{OD}_{600}$ of 0.1. Wells of a 96-well flat-bottom cell culture plate (Greiner Bio-one) were filled with $120 \mu \mathrm{l}$ LB media, and $20 \mu \mathrm{l}$ of each cell culture was added to each respective well. The weak acids tested were docusate, $n$-lauroyl sarcosine, and probenecid. Probenecid was dissolved into dimethyl sulfoxide (DMSO) prior to use. Docusate was tested as six two-fold serial dilutions from $2.5 \mathrm{mg}$ to $0.039 \mathrm{mg}$ per $\mathrm{mL} \mathrm{LB}$ broth, and $n$-lauroylsarcosine and probenecid were tested at 10 -fold higher concentrations than docusate. Wells that did not receive any weak acids served as no-drug controls. The assay with probenecid contained additional no-drug controls where the strains were grown with DMSO. The wells containing $n$-lauroylsarcosine and probenecid were overlaid with $50 \mu \mathrm{l}$ mineral oil, and the plates were incubated at $37{ }^{\circ} \mathrm{C}$ and shaking at medium intensity. Growth measurements were recorded at $\mathrm{OD}_{595}$ every hour for $24 \mathrm{~h}$. Plates with docusate were covered with lids and wrapped in parafilm and incubated at $37^{\circ} \mathrm{C}$ and shaking at $7 \times g$ in an orbital shaker for $24 \mathrm{~h}$. Growth measurements were taken every 4-h using an iMark plate reader (BioRad). Each strain was tested in technical duplicates, and the experiment was independently repeated eight times for docusate, three times for probenecid, and six times for $n$-lauroylsarcosine.

\section{Statistical analysis}

For all statistical analyses, GraphPad Prism version 7.0 for Windows (GraphPad Software, La Jolla California USA) was used. A two-way ANOVA and Dunnett's multiple-comparison tests were employed on all data sets that were previously confirmed to be normally distributed by D'Agostino-Pearson normality test. All results were compared to the wild-type, and $p \leq 0.001$ was considered statistically significant unless otherwise noted.

\section{Abbreviated summary}

Pseudomonas aeruginosa's secondary sodium pump Mrp is crucial for survival, growth, biofilm formation, and swarming motility of the organism in challenging environmental conditions (high $\mathrm{pH}$ and sodium). In contrast, lack of the primary sodium pump Complex I had some consistent impact on $P$. aeruginosa's growth, but those changes were independent of $\mathrm{pH}$ and sodium concentration. Additionally, the primary sodium pump NQR and the secondary sodium pumps $\mathrm{NhP}$ and $\mathrm{NhaB}$ played only minimal or insignificant roles.

\section{Abbreviations \\ $w / v$ : weight per volume}

\section{Acknowledgements}

We are grateful to Dr. Martin Schuster for providing the strains, Dr. Julia Steuber for the testing of our LBB-residual cation concentrations and her useful insights, and Dr. Alisha Aagesen for early work on this project.

Studies involving plants must include a statement specifying the local, national or international guidelines and legislation and the required or appropriate permissions and/or licenses for the study Not applicable 


\section{Authors' contributions}

CBS designed experiments, performed work related to growth assays and swarming motility, formatted all the graphs, and wrote the manuscript. KHTH designed the experiments, was responsible for acquiring and analyzing all the data, and drafted parts of and revised the manuscript. CCH conceived and designed the study, supervised the research, and drafted parts of and revised the manuscript. All authors read and approved the final manuscript.

\section{Authors' information}

Not applicable

\section{Funding}

Internal awards by the Office of Undergraduate Education and the Carlson College of Veterinary Medicine provided financial support for this work to Kelli H.T. Hoang.

\section{Availability of data and materials}

The datasets used and/or analyzed during the current study are available from the corresponding author on reasonable request.

\section{Ethics approval and consent to participate}

Not applicable

\section{Consent for publication}

Not applicable

\section{Competing interests}

The authors declare that they have no competing interests.

\section{Author details}

'Department of Biomedical Sciences, Carlson College of Veterinary Medicine, Oregon State University, Corvallis, OR 97331, USA. ${ }^{2}$ College of Pharmacy, Oregon State University, Corvallis, OR 97331, USA.

\section{Received: 8 November 2019 Accepted: 6 January 2020}

\section{Published online: 03 February 2020}

\section{References}

1. Aagesen AM, Schubiger CB, Hobson EC, Dibrov P, Hase CC (2016) Effects of chromosomal deletion of the operon encoding the multiple resistance and pH-related antiporter in Vibrio cholerae. Microbiology 162:2147-2158

2. Arai $H$ (2011) Regulation and function of versatile aerobic and anaerobic respiratory metabolism in Pseudomonas aeruginosa. Front Microbio/ 2:103

3. Barquera B, Hellwig P, Zhou W, Morgan JE, Hase CC, Gosink KK et a (2002) Purification and characterization of the recombinant $\mathrm{Na}(+$ )-translocating NADH:quinone oxidoreductase from Vibrio cholerae. Biochemistry 41:3781-3789

4. Beattie P, Tan K, Bourne RM, Leach D, Rich PR, Ward FB (1994) Cloning and sequencing of four structural genes for the $\mathrm{Na}(+)$-translocating $\mathrm{NADH}$ ubiquinone oxidoreductase of Vibrio alginolyticus. FEBS Lett 356:333-338

5. Belas R (2014) Biofilms, flagella, and mechanosensing of surfaces by bacteria. Trends Microbiol 22:517-527

6. Bertsova YV, Bogachev AV (2004) The origin of the sodium-dependent $\mathrm{NADH}$ oxidation by the respiratory chain of Klebsiella pneumoniae. FEBS Lett 563:207-212

7. Bren A, Hart Y, Dekel E, Koster D, Alon U (2013) The last generation of bacterial growth in limiting nutrient. BMC Syst Biol 7:27

8. Costerton JW, Stewart PS, Greenberg EP (1999) Bacterial biofilms: a common cause of persistent infections. Science 284:1318-1322

9. Dibrov P, Dibrov E, Maddaford TG, Kenneth M, Nelson J, Resch C, Pierce GN (2017a) Development of a novel rationally designed antibiotic to inhibit a nontraditional bacterial target. Can J Physiol Pharmacol 95:595-603

10. Dibrov P, Dibrov E, Pierce GN (2017b) Na+-NQR (Na+-translocating NADH: ubiquinone oxidoreductase) as a novel target for antibiotics. FEMS Microbiol Rev 41:653-671

11. Donlan RM, Costerton JW (2002) Biofilms: survival mechanisms of clinically relevant microorganisms. Clin Microbiol Rev 15:167-193

12. Doyle TB, Hawkins AC, McCarter LL (2004) The complex flagellar torque generator of Pseudomonas aeruginosa. J Bacteriol 186:6341-6350

13. Dzioba-Winogrodzki J, Winogrodzki O, Krulwich TA, Boin MA, Hase CC, Dibrov P (2009) The Vibrio cholerae Mrp system: cation/proton antiport properties and enhancement of bile salt resistance in a heterologous host. $J$ Mol Microbiol Biotechnol 16:176-186

14. Friedland, G., Jantz, K., and Rojas, R. (2005) SIOX: Simple interactive object extraction in still images. In Proceedings - Seventh IEEE International Symposium on Multimedia, ISM 2005. .

15. Gellatly SL, Hancock REW (2013) Pseudomonas aeruginosa : new insights into pathogenesis and host defenses. Pathog Dis 67:159-173 https://doi.org/ 10.1111/2049-632X.12033

16. Gemperli AC, Dimroth P, Steuber J (2002) The respiratory complex I (NDH I) from Klebsiella pneumoniae, a sodium pump. J Biol Chem 277:33811-33817

17. Gemperli AC, Schaffitzel C, Jakob C, Steuber J (2007) Transport of $\mathrm{Na}(+)$ and K (+) by an antiporter-related subunit from the Escherichia coli NADH dehydrogenase produced in Saccharomyces cerevisiae. Arch Microbiol 188:509-521

18. Hase CC, Mekalanos JJ (1999) Effects of changes in membrane sodium flux on virulence gene expression in Vibrio cholerae. Proc Natl Acad Sci U S A 96: 3183-3187

19. Hayashi $M$, Hirai $K$, Unemoto $T$ (1995) Sequencing and the alignment of structural genes in the nqr operon encoding the $\mathrm{Na}(+)$-translocating $\mathrm{NADH}-$ quinone reductase from Vibrio alginolyticus. FEBS Lett 363:75-77

20. Hayashi M, Nakayama Y, Unemoto T (1996) Existence of Na+-translocating $\mathrm{NADH}$-quinone reductase in Haemophilus influenzae. FEBS Lett 381:174-176

21. Herz K, Vimont S, Padan E, Berche P (2003) Roles of NhaA, NhaB, and NhaD $\mathrm{Na}+/ \mathrm{H}+$ antiporters in survival of Vibrio cholerae in a saline environment. J Bacteriol 185:1236-1244

22. Ito M, Morino M, Krulwich TA (2017) Mrp antiporters have important roles in diverse bacteria and archaea. Front Microbiol 8:2325 https://www.frontiersin. org/article/10.3389/fmicb.2017.02325

23. Juarez O, Shea ME, Makhatadze Gl, Barquera B (2011) The role and specificity of the catalytic and regulatory cation-binding sites of the $\mathrm{Na+}$ pumping NADH:quinone oxidoreductase from Vibrio cholerae. J Biol Chem 286:26383-26390

24. Kearns DB (2010) A field guide to bacterial swarming motility. Nat Rev Microbiol 8:634-644

25. Kohler T, Curty LK, Barja F, van Delden C, Pechere JC (2000) Swarming of Pseudomonas aeruginosa is dependent on cell-to-cell signaling and requires flagella and pili. J Bacteriol 182:5990-5996

26. Kosono S, Haga K, Tomizawa R, Kajiyama Y, Hatano K, Takeda S et al (2005) Characterization of a multigene-encoded sodium/hydrogen antiporter (sha) from Pseudomonas aeruginosa: its involvement in pathogenesis. J Bacteriol 187:5242-5248

27. Kostyrko VA, Bertsova Y, Serebryakova MV, Baykov AA, Bogachev AV (2015) NgrM (DUF539) Protein is required for maturation of bacterial $\mathrm{Na+}$ translocating NADH:quinone oxidoreductase. J Bacteriol 198:655-663

28. Kuroda T, Fujita N, Utsugi J, Kuroda M, Mizushima T, Tsuchiya T (2004) A major Li(+) extrusion system NhaB of Pseudomonas aeruginosa : comparison with the major $\mathrm{Na}(+)$ extrusion system NhaP. Microbiol Immunol 48:243-250

29. Lamas Ferreiro JL, Alvarez Otero J, Gonzalez Gonzalez L, Novoa Lamazares L, Arca Blanco A, Bermudez Sanjurjo JR et al (2017) Pseudomonas aeruginosa urinary tract infections in hospitalized patients: mortality and prognostic factors. PLoS One 12:e0178178

30. Liberati NT, Urbach JM, Miyata S, Lee DG, Drenkard E, Wu G et al (2006) An ordered, nonredundant library of Pseudomonas aeruginosa strain PA14 transposon insertion mutants. Proc Natl Acad Sci U S A 103:2833-2838

31. Mathiesen C, Hägerhäll C (2003) The "antiporter module" of respiratory chain Complex I includes the MrpC/NuoK subunit - a revision of the modular evolution scheme. FEBS Lett 549:7-13

32. McCarter LL (2004) Dual flagellar systems enable motility under different circumstances. J Mol Microbiol Biotechnol 7:18-29

33. Morino M, Ogoda S, Krulwich TA, Ito M (2017) Differences in the phenotypic effects of mutations in homologous MrpA and MrpD subunits of the multisubunit Mrp-type $\mathrm{Na}(+) / H(+)$ antiporter. Extremophiles 21:51-64

34. Overhage J, Lewenza S, Marr AK, Hancock REW (2007) Identification of genes involved in swarming motility using a Pseudomonas aeruginosa PAO1 mini-Tn5-lux mutant library. J Bacterio/ 189:2164-2169

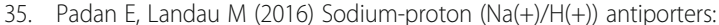
properties and roles in health and disease. Met lons Life Sci 16:391-458

36. Padan E, Venturi $M$, Gerchman $Y$, Dover N (2001) Na(+)/H(+) antiporters. Biochim Biophys Acta 1505:144-157

37. Raba DA, Rosas-Lemus M, Menzer WM, Li C, Fang X, Liang P et al (2018) Characterization of the Pseudomonas aeruginosa NQR complex, a bacterial 
proton pump with roles in autopoisoning resistance. J Biol Chem 293: $15664-15677$

38. Rasband, W. 1997-2018 (2018) ImageJ. https://imagej.nih.gov/ij/.

39. Rashid MH, Kornberg A (2000) Inorganic polyphosphate is needed for swimming, swarming, and twitching motilities of Pseudomonas aeruginosa. Proc Natl Acad Sci U S A 97:4885-4890

40. Reyes-Prieto A, Barquera B, Juarez O (2014) Origin and evolution of the sodium -pumping NADH: ubiquinone oxidoreductase. PLoS One 9:e96696

41. Sakuma T, Yamada N, Saito H, Kakegawa T, Kobayashi H (1998) pH dependence of the function of sodium ion extrusion systems in Escherichia coli. Biochim Biophys Acta 1363:231-237

42. Shrout JD, Chopp DL, Just CL, Hentzer M, Givskov M, Parsek MR (2006) The impact of quorum sensing and swarming motility on Pseudomonas aeruginosa biofilm formation is nutritionally conditional. Mol Microbiol 62: 1264-1277

43. Steuber J (2003) The C-terminally truncated NuoL subunit (ND5 homologue) of the Na+-dependent complex I from Escherichia coli transports Na+. J Biol Chem 278:26817-26822

44. Steuber J, Schmid C, Rufibach M, Dimroth P (2000) Na+ translocation by complex I (NADH:quinone oxidoreductase) of Escherichia coli. Mol Microbiol 35:428-434

45. Swartz TH, Ikewada S, Ishikawa O, Ito M, Krulwich TA (2005) The Mrp system: a giant among monovalent cation/proton antiporters? Extremophiles 9:345354 https://doi.org/10.1007/s00792-005-0451-6

46. Tremblay J, Deziel E (2008) Improving the reproducibility of Pseudomonas aeruginosa swarming motility assays. J Basic Microbiol 48:509-515

47. Utsugi J, Inaba K, Kuroda T, Tsuda M, Tsuchiya T (1998) Cloning and sequencing of a novel $\mathrm{Na}+/ \mathrm{H}+$ antiporter gene from Pseudomonas aeruginosa. Biochim Biophys Acta 1398:330-334

48. Verstraeten N, Braeken K, Debkumari B, Fauvart M, Fransaer J, Vermant J, Michiels J (2008) Living on a surface: swarming and biofilm formation. Trends Microbiol 16:496-506

49. Wakabayashi S, Shigekawa M, Pouyssegur J (1997) Molecular physiology of vertebrate $\mathrm{Na}+/ \mathrm{H}+$ exchangers. Physiol Rev 77:51-74

50. Williams HD, Zlosnik JEA, Ryall B (2007) Oxygen, cyanide and energy generation in the cystic fibrosis pathogen Pseudomonas aeruginosa. Adv Microb Physiol 52:1-71

51. Zhou, W., Bertsova, Y. V, Feng, B., Tsatsos, P., Verkhovskaya, M.L., Gennis, R.B., et al. (1999) Sequencing and preliminary characterization of the Na+translocating NADH:ubiquinone oxidoreductase from Vibrio harveyi. Biochemistry 38: 16246-16252.

\section{Publisher's Note}

Springer Nature remains neutral with regard to jurisdictional claims in published maps and institutional affiliations.

\section{Submit your manuscript to a SpringerOpen ${ }^{\circ}$ journal and benefit from:}

- Convenient online submission

- Rigorous peer review

- Open access: articles freely available online

- High visibility within the field

- Retaining the copyright to your article

Submit your next manuscript at $\boldsymbol{\nabla}$ springeropen.com 